\title{
Synchronous Distance Education: Enhancing Speaking Skills via Internet-based Real
}

\section{Time Technology}

\author{
Yuping Wang \\ School of Languages and Linguistics \\ Griffith University, Australia
}

\author{
Chengzheng Sun \\ School of Computing and \\ Information Technology \\ Griffith University, Australia.
}

\begin{abstract}
This paper reports an investigation into one of the most urgent problems facing distance language education - the problem of lack of exposure to speaking practice in the target language. The Open Learning Chinese Program taught at Griffith University is used as a case study. Following a discussion of issues relating to distance education for languages, such as the indispensability of technology to learning languages in a distance mode, and the importance of communicative competence, this paper moves on to an examination of the capabilities of Internetbased real time technology. Two major indications can be generated from this research: real time technology can help solve the problem of insufficient exposure to speaking practice, and a historical convergence of distance and traditional campus-based education toward a networked education can be expected.
\end{abstract}

\section{Introduction}

The point of departure for this paper is the identification of the problems associated with learning languages in distance mode. It proceeds to discuss possible solutions to the major problem identified, i.e., the problem of lack of exposure to speaking practice. This discussion will function as a basis for predicting the future of distance language education. The paper employs the Open Learning
Chinese Program offered at Griffith University as a case study.

The main focus of this research is thus, through an investigation of the Open Learning Chinese program at Griffith University, to gain an insight into the following critical issues confronting language learning at a distance. What is the most urgent problem with learning languages in distance mode? Is Computer Mediated Conference (CMC) using real time technology effective in tackling this problem? What is the future of this mode of language learning and teaching? In a word, what are the major implications of advanced computer technology to distance education for languages? While more and more research on the use of CMC to improve student's writing and reading comprehension is being published, there is a paucity of systematic findings on the effectiveness of $\mathrm{CMC}$ in improving student's speaking ability. It is hoped that this paper will help fill this gap.

\section{Identification of problems}

While distance learning is applauded by many for its flexibility in delivery, minimal teacher contact etc, as far as learning languages in distance mode is concerned, this flexibility has been achieved at the expense of quality. Involvement with the Open Learning Chinese program at 
Griffith University as a teacher and course developer has provided us with first hand knowledge about the joy and frustration of those distance language learners. They are delighted to have the opportunity of learning a language wherever and whenever they want, and without having to attend a class at a prescribed time. Qualifying the positive feelings is the frustration they often feel when they are learning to communicate but there is no one to turn to for communication in the target language. It would not be an exaggeration to say that physical distance prevents effective language learning and teaching from happening. In other words, without the adequate support of technology, the distance mode of delivery, particularly in the case of language learning and teaching, has fatal deficiencies.

\subsection{The case of the Open Learning Chinese Program at Griffith University}

In the present structure of course delivery, the task of practising speaking is largely left to the student with little academic support and no spontaneous feedback at all. As the only measure to check students' progress in speaking, one section of the assignments usually requires the students to record a few sentences or passages onto a tape. The tape is then submitted together with their written assignments. After listening to the tape, the teacher is only able to point out to the students which sound was pronounced wrongly, and why it was wrong. For example, the teacher would write comments to warn the student that the ma sound with the first tone was incorrectly pronounced in the fourth tone. However, this does not ensure that the student will know how to pronounce the ma sound in the fourth tone after reading the teacher's detailed notes. During our years teaching with the program, we often found ourselves recording the same passage for some of the students after listening to their recordings and asking the students to listen to our recording, in the hope that the students would notice the difference between the correct pronunciation and theirs This is an enormous task for the teacher if there is a great number of students. Even so, there is no way to guarantee that the students listen to our recording, let alone ascertain that they can decipher the difference between the correct and incorrect pronunciations. This problem is particularly acute in the case of learning Chinese language, for the simple reason that Chinese is a tonal language. Of the many difficulties encountered by beginners of Chinese language, mastery of the tones in Chinese is always a daunting experience. This difficulty is ascribable to the fact that one has to pronounce the sound in a certain pitch, known as tone. There are 4 tones, or 5 including the neutral one. "The critical importance of tonal accuracy in the development of spoken competence in Chinese cannot be underestimated" [5]. This is because a slight variation in the tone can represent a totally different character with a different meaning. For example, ma with the first tone can mean "mother" (妈) while mă with the third tone can mean "horse" $\left(I_{J}\right)$. Learners have to hear and watch the sound being pronounced to be able to ascertain the subtle differences between the syllables.

Assuming that our students can attain perfect pronunciation and tones, and can remember all the useful phrases and sentences after studying assiduously with books and tapes, spontaneous conversation between the learners and teacher, and among the learners themselves, still cannot possibly be generated. Consequently, students still find themselves lacking a simple reflex answer to a very basic sentence that they have already practised many times. This is because apart from being daunted by the speaker's unfamiliar accent and intonation, our students are not used to and have not acquired the skills for communicating in the target language. Students' communicative skills are almost non-existent.

Clearly the training of speaking skills is an integral part of language acquisition and plays a crucial role in effective language learning. In other words, a highly interactive and communicative learning environment is conducive to successful language learning. Unfortunately this is precisely where distance language education falls short. To create such a learning environment should be the top priority in the distance language professionals' endeavors. This paper 
argues that Chinese language is not suffering in isolation. In fact, lack of interactive speaking practice is an intrinsic problem for all the distance mode of learning languages. Naturally, it has been an ongoing effort by distance language professionals to find ways to solve this problem.

\section{Solutions to the problem}

\subsection{Technologies used in distance language education: four generations}

As discussed above, the problem of lack of speaking training came to the fore with the inception of the distance mode of learning languages. In the face-to-face mode, presentation of subject matter is essentially a process of interactive communication. Unfortunately, in distance education, due to the barrier of physical distance, interactivity has been divorced from the presentation of subject matter. In their constant search for solutions, it is no coincidence that language professionals have turned to technology for help. Assisted by technology, the scope and content of interaction have been continually enriched. In other words, in different phases of distance education, interaction entails different content and meaning.

Distance education for languages has a history of more than a century. The development of distance language education can be divided into four generations [7]. Different from campus-based education, technologies used in each of the generations in distance education are not just supplementary, but also a necessity. This is a distinctive feature of technologies used in distance education because distance learning presents a different set of challenges to both teachers and learners from that of the traditional classroom. Without the aid of technology, distance education would be impossible. The use of technology such as the postal system in the first generation, telephone, video, radio and television broadcasting in the second generation, and computer in the third generation, has greatly improved the quality of distance education by providing more direct and efficient communication and interaction between the learner and instructor.

The 1990s witnessed revolutionary changes in distance education for languages when Internet-based real time technology came onto the educational scene. For the first time in history, the problem of insufficient exposure to spontaneous speaking practice appears to be solvable, as will be discussed below.

It can be concluded that technologies used in distance education have evolved from single-medium to multi-media, and thus changing distance education for languages from off-line to on-line and from asynchronous to synchronous. This process pushes distance education to advance to a higher level of achievement. However it should be noted that there is no clear-cut set of technologies used in each generation. Telephones and videos, even audiocassettes, are still used in the third or even the fourth generation together with internet-based real time technology.

\subsection{Internet-based real time technology provides possible solutions}

This study explores the use of real time technology, such as Net Meeting and IPhone in conducting real-time tutorials in distance learning mode. Aided by the abovementioned real time technology, languages being learnt have "come alive" [1]. Learners can have spontaneous conversation in the target language with other learners, teachers and even native speakers of the target language. For example, through desktop video conferencing or Internet telephoning, students can be exposed to a virtual classroom situation, in which they can hear the target language being spoken, watch the body language of the speaker, and use the target language to communicate and solve problems. Similarly, students can perform a role-play in the target language with other students. As a result, language learning is now happening in a social context and becoming more meaningful than ever. Students can be exposed to a variety of dialects and accents in the target language and have an opportunity to develop language spontaneity and reflexiveness. In such a learning environment, better 
retention can be expected.

This is a most significant improvement in that it caters for the special needs of distance language learners, i.e. the need to cultivate listening and speaking skills. Internet-based real time technology effectively facilitates the development of such skills and thus removes the constraints placed by physical distance on learning languages at a distance. At the same time, it provides both an interaction similar to that of face-to-face communication, and a virtual social context in which the use of the target language occurs most naturally. Thus real time technology brings the distance learner into the real world, both in the sense of reality and in the realm of virtual learning environment.

\subsection{The case of the Open Learning Chinese program at Griffith University}

We are planning to develop and manage a Web site for the existing Open Learning Chinese program at Griffith University. This Web site will differ from existing Web based language courseware in that it will not only present course information in a manner conventionally used on the Web, but will also address the above-discussed problem, i.e., the lack of speaking exposure to the target language. This web-based learning environment will provide an interface for communicative tasks, such as listening and speaking, and students' interaction with the teacher and with their peers, etc, through the use of advanced real-time technologies such as Net Meeting, and/or I-Phone.

Through real time technology, a weekly real time tutorial on listening and speaking training will be offered to the students, in which students and teacher can see and talk to each other as in a real classroom. For the beginners of the program, the teacher will take this opportunity to demonstrate how to pronounce certain sounds, how to move the lips, how to position the tongue etc. At the same time, real time technology can ensure more exposure to speaking tasks for advanced learners who can carry on real time conversations in Chinese with the teacher and their fellow students.
It is hoped that the use of real time technology will improve students' speaking skills and promote student's motivation to learn the language.

\section{Future direction of distance education}

Drawing on the results of this research, this paper predicts a total re-conceptualization of distance education. With the advancement in network technology and its extensive use in education, the distinction between distance and campus-based education is becoming more and more blurred. Globalization now has a two-fold implication for education: the globalization of distance learning and the globalization of education as a whole. In response to this trend, the late 1990s heard repeated calls to "reengineer", "redefine" and "reshape" modern education. In 1996, Dede's speculation about how emerging technologies may reshape distance as well as face-to-face education generated a heated dialogue among the reviewers of his article. He depicts a new education system called distributed learning, consisting of knowledge webs, virtual communities and shared synthetic environments [3]. Today, this speculation has been more widely accepted as the future of education. Scholars are even announcing a historical convergence of distance education and traditional campus-based education toward a networked education. This announcement is based on research into the potentials of network technologies $[3,4$, $2,6]$. The above-discussed changes indicate a whole new kind of distance education system with fundamentally improved capability will soon emerge.

\section{Conclusion}

The significance of this paper lies in the efforts to identify one of the most urgent and serious problems with distance language education, i.e. the problem of lack of spontaneous interaction. In the attempt to find solutions to this problem, this paper reviews, for the distance language profession, the roles played by technology in the evolution of distance language education. It further depicts the 
availability and capabilities of today's educational technology, real time technology in particular. All these discussions then lead to a prediction of the future of modern education.

The results of this research show that the technology to support synchronous learning is in place, and it is improving and expanding rapidly. Among the innovations and changes brought about by computer technology, the change of distance language education from asynchronous to synchronous through the use of Internet-based real time technology is the most significant one. In a real time learning environment, the effect of physical distance on the interaction between learner and instructor can be reduced to the minimum. In other words, Internet-based real time technology facilitates an interaction nearly as effective as that of face-to-face. Such interaction is a real time interaction with spontaneity as its defining feature, and is conducive to language learning. On the other hand, Internetbased real time technology provides learners with plentiful opportunities to engage in meaningful language learning. This paper concludes that in the $21^{\text {st }}$ century, technology is not simply an add-on medium of delivery as were those technologies employed before; rather, "it touches the very substance of the university" [6]. It seems safe to conclude that advanced technology is leading modern education to an immersion of learning at a distance and on campus. The word 'distance' and the physical distance in education may disappear all together. It is hoped that the results of this research will help the distance language educator and learner better understand the capabilities and potential of distance language education and what technologies can be utilized to further improve the distance mode of language learning.

\section{References}

[1] D. Chun, "Using computer networks to facilitate the acquisition of interactive competence", System, 22 (1): 17-31, 1994.

[2] S. Cunningham, S. Tapsall, Y. Ryan, L. Stedman, K. Bagdon, \& T. Flew, New Media and Borderless Education: A Review of the Convergence between Global Media Networks and Higher Education Provision, Commonwealth of Australia, 1998

[3] C. Dede, "The evolution of distance education: emerging technologies and distributed learning", The American Journal of Distance Education, 10 (2): 4-36, 1996

[4] J.W. Hall, "Leadership in accreditation and networked learning", The American Journal of Distance Education. 12 (2): 5-15, 1998.

[5] S. McGinnis, "Tonal Spelling versus diacritics for teaching pronunciation of Mandarin Chinese", The Modern Language Journal, 81, ii: 228-236, 1997

[6] D. Tsichritzis, "Reengineering the University", Communications of the ACM, 42 (6): 93-100, 1999

[7] Y. Wang \& C. Sun, "Internet-based real time language education: towards a fourth generation distance education", CALCO, forthcoming, 2000 